\title{
Tratamiento de la obstrucción neoplásica del colon mediante la inserción endoscópica de prótesis metálicas autoexpandibles
}

\author{
J. GARCÍA-CANO, J. A. GONZÁLEZ MARTÍN, E. REDONDO-CEREZO, \\ J. MORILLAS ARIÑO, J. I. PÉREZ GARCÍA, M. G. PÉREZ VIGARA, C. J. GÓMEZ \\ RUIZ, A. PÉREZ SOLA
}

Sección de Aparato Digestivo. Hospital Virgen de la Luz. Cuenca

TREATMENT OF MALIGNANT COLORECTAL OBSTRUCTION BY MEANS OF ENDOSCOPIC INSERTION OF SELF-EXPANDABLE METALLIC STENTS

\section{RESUMEN}

Antecedentes y objetivo: Las prótesis metálicas autoexpandibles están siendo utilizadas, cada vez con más frecuencia, para tratar la obstrucción de diversos segmentos del tracto digestivo y de la vía biliar. Presentamos la experiencia en nuestro centro de la resolución inicial de la obstrucción del colon, de origen tumoral, mediante este tipo de prótesis.

Pacientes y métodos: Durante un período de 18 meses, se intentó resolver inicialmente la obstrucción neoplásica del colon (recto, sigma y descendente) en 13 pacientes, mediante prótesis (Wallstent enteral sin recubrir) insertadas endoscópicamente. En diez ocasiones se utilizó endoscopia y fluoroscopia, y en tres, sólo endoscopia.

Resultados: La obstrucción se resolvió en 12 de los 13 pacientes $(92,3 \%)$ tras la inserción correcta de las prótesis. Todos los procedimientos exclusivamente endoscópicos tuvieron éxito. En seis (50\%) pacientes con neoplasias rectosigmoideas se realizó posteriormente cirugía programada. En los seis restantes (una paciente con cáncer de ovario y cinco con adenocarcinomas de colon), la prótesis se consideró como tratamiento paliativo definitivo. En dos de estos pacientes la prótesis migró, extrayéndose endoscópicamente. Sólo uno de ellos necesitó otra prótesis. En otra paciente, en el grupo paliativo, se desarrolló una fistula tumoral colo-vesical dentro de la prótesis, y se trató mediante la inserción de otra prótesis, esofágica recubierta (Ultraflex), dentro de la anterior. No hubo otras complicaciones ni mortalidad relacionada con el procedimiento endoscópico.

Conclusiones: Las prótesis metálicas autoexpandibles podrían considerarse, en general, como el tratamiento inicial de la obstrucción neoplásica colónica a nivel de recto-sigma-descendente.

PALABRAS CLAVE: Obstrucción tumoral del colon. Prótesis metálicas autoexpandibles. Intervencionismo endoscópico digestivo. Endoscopia terapéutica digestiva

\section{ABSTRACT}

Background and aim: Self-expandable metallic stents are being used increasingly to treat the obstruction of different segments of the digestive tract and biliary tree. We present our centre experience on the initial resolution of malignant colorectal obstruction by means of this type of stents.

Patients and methods: During a 18-month period, 13 patients patients suffering from malignant obstruction at the level of rectum, sigmoid or descending colon tried to be initially treated by means of endoscopic insertion of stents (non covered enteral Wallstents). Ten procedures were performed with both endoscopy and fluroscopy and three with only endoscopy.

Results: In 12 of the 13 patients (92,3\%) the obstruction was solved by means of correct stent insertion. All the exclusively endoscopic procedures (without fluoroscopy) were successful. Six (50\%) patients with tumours at the rectosigmoid underwent later scheduled surgery. In the remaining six ones (a patient with an ovarian carcinoma and five with colonic adenocarcinoma) the stents were considered to be a palliative definitive treatment. Stent migration was observed in two of these patients and both were extracted endoscopically. Only one patient needed to have another stent inserted. A tumoural colo-vesical fistula developed in another patient in the palliative group, inside the previous inserted stent, and was treated by coaxial insertion of an esophageal Ultraflex. There were no other complications or mortality related to the endoscopic procedures.

Conclusions: Self-expandable metallic stents might be considered, in general, as the initial treatment for the malignant obstruction at the level of rectum, sigmoid and descending colon.

KEY WORDS: Malignant colorectal obstruction. Self-expandable metal stents. Interventional digestive endoscopy. Therapeutic digestive endoscopy.

García-Cano J, González Martín JA, Redondo-Cerezo E, Morillas Ariño J, Pérez García JI, Pérez, Vigara MG, Gómez Ruiz CJ, Pérez Sola A. Tratamiento de la obstrucción neoplasica del colon mediante la inserción endoscópica de prótesis metálicas autoexpandibles. An Med Interna (Madrid) 2003; 20: 515-520.

\section{INTRODUCCIÓN}

La obstrucción es un fenómeno patológico común a todos los órganos tubulares del organismo. Las consecuencias fisiopatológicas varian, lógicamente, según el lugar anatómico en que se produzca el fenómeno obstructivo.
Desde hace años, el desarrollo de la tecnología ha permitido utilizar distintos materiales para fabricar lo que se denominan, prótesis metálicas autoexpandibles (PMA). Las PMA son unos tubos cilíndricos, en forma de malla, de distintas longitudes y anchuras, que tienen la característica de poderse insertar plegadas. Esta propiedad permite atravesar estenosis muy

Trabajo aceptado: 6 de mayo de 2003 
importantes sin necesidad de dilatarlas previamente. Una vez dentro de la zona obstruida, mediante un mecanismo de liberación, las PMA se abren, consiguiendo un diámetro suficiente para recanalizar la zona estenosada. Las PMA se denominan también, especialmente en la literatura científica escrita en inglés, stents. La palabra proviene del apellido (Stent) de uno de los ingenieros que más trabajaron, inicialmente, en el estudio de las propiedades de ciertos materiales metálicos para recuperar su forma original (autoexpansión) tras ser plegados. De la palabra se deriva también la acción de insertar una PMA o stenting.

Las PMA -con modificaciones según el lugar anatómicose utilizan para recanalizar la obstrucción de organos tubulares macroscópicos como las coronarias (1), la tráquea (2) o gran parte del tubo digestivo y de la vía biliar (3).

En 1991 se describió, por primera vez, la utilización de una PMA para paliar la obstrucción colónica aguda (4). Desde entonces ha habido mejoras tecnológicas en las PMA. Han aparecido modelos específicamente diseñados para recanalizar tramos enterales y, también, ha aumentado su utilización por parte de endoscopistas y radiólogos (5-8), con muy buenos resultados.

Presentamos la experiencia en nuestro centro, de la resolución inicial de la obstrucción colónica aguda, de origen tumoral, mediante la inserción endoscópica de PMA.

\section{PACIENTES Y MÉTODOS}

Se han revisado, de forma retrospectiva, los pacientes cuya obstrucción colónica intentó resolverse mediante la inserción de PMA durante un periodo de 18 meses.

En nuestro centro, en general, ante la posibilidad de una obstrucción colónica diagnosticada mediante la historia clínica, exploración física y radiología simple de abdomen, se realiza una colonoscopia de urgencia. En ella intenta insuflarse la mínima cantidad de aire posible, utilizando la irrigación de agua para ayudar a distender la luz del colon y hacer progresar el endoscopio. Una vez diagnosticada la obstrucción de origen tumoral se toman habitualmente biopsias, y, de acuerdo con el equipo de Cirugía, se ha intentado la resolución de la misma por medio de PMA.

El paciente y el equipo endoscópico se trasladaban a una sala de radiología con posibilidad de fluoroscopia. Se utilizaron colonoscopios con canal de trabajo terapéutico. Las intervenciones endoscópicas han sido realizadas por dos gastroenterólogos con experiencia en intervencionismo endoscópico (JGC y JAGM). Una vez situado el endoscopio en la parte más distal del tumor (raras veces puede atravesarse la obstrucción), se intentó pasar una guía teflonada de 0,035 pulgadas de diámetro $(0,875 \mathrm{~mm})$ y con punta hidrofílica atraumática (Jagwire superstiff, Boston Scientific/Microvasive), a través del canal de trabajo del colonoscopio con la intención de atravesar ("enhebrar") todo el segmento estenosado (Fig. 1). Este es el punto más determinante para la correcta inserción de PMA.

La comprobación de que la guía ha atravesado la obstrucción y está en colon normal se realiza mediante fluroscopia. En los casos de PMA colocadas exclusivamente con endoscopia (sin radiología), se consideraba que la guía había atravesado toda la obstrucción, por medio de la sensación táctil del endoscopista de que la guía pasaba libre-

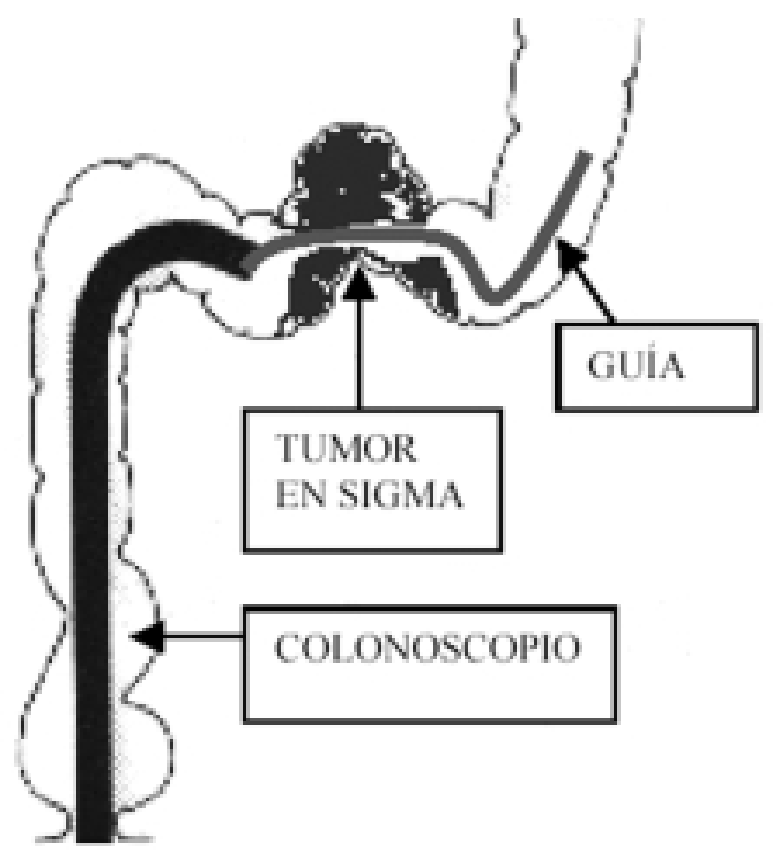

Fig. 1. Esquema en el que se muestra el paso de la guía desde el colonoscopio a través del tumor. Este es el punto más determinante para la inserción correcta de las prótesis. Sobre la guía y a través del canal de trabajo del endoscopio se hace avanzar la prótesis plegada, que posteriormente, una vez colocada dentro de la estenosis, se expande.

mente y por la ausencia de imágenes, en la pantalla del videoendoscopio, de que la guía se volviera. La sensación táctil es similar, por ejemplo, a la facilidad con que la guía metálica debe pasar tras canalizar correctamente una vena subclavia.

En las PMA insertadas en la sala de radiología, a través de la guía se introducía un catéter biliar de los utilizados en la Colangiopancreatografía retrógrada endoscópica (CPRE) y por medio de la inyección de contraste se dibujaba la obstrucción. Se retiraba después el catéter y, siempre sobre la guía, se insertaba la prótesis más adecuada. Una vez que la PMA se encontraba dentro de la estenosis (dejando unos centímetros a ambos lados de la misma) comenzaba a desplegarse gradualmente. Durante el periodo de liberación, el endoscopista debe corregir continuamente el acortamiento que sufren las prótesis al pasar de su situación plegada a completamente expandidas. Habitualmente se han utilizado PMA de tipo Wallsten enteral (Boston Scientific/Microvasive). Esta PMA tiene, una vez expandida del todo, una longitud de 6 ó $9 \mathrm{~cm}$ y un diámetro de 2,2 $\mathrm{cm}$. Su malla no está recubierta. Esto sirve para que se ancle bien en el tumor (que penetra por los orificios de la malla), aunque con el tiempo se obstruye. En los casos en que las PMA se introdujeron sólo con endoscopia, al desconocer la longitud de la estenosis, se colocó siempre la de $9 \mathrm{~cm}$ de longitud.

Tras la inserción de la prótesis, el enfermo se mantuvo ingresado para completar el estudio. En los días sucesivos se comprobó su mejoría clínica y se vigiló la existencia de posibles complicaciones. 


\section{TABLA I}

CARACTERÍSTICAS DE LOS PACIENTES CUYA OBSTRUCCIÓN COLÓNICA TUMORAL SE TRATÓ MEDIANTE PRÓTESIS METÁLICAS AUTOEXPANDIBLES

\begin{tabular}{llllll}
\hline Paciente Edad Sexo & $\begin{array}{l}\text { Nivel dela } \\
\text { obstrucción } \\
\text { neoplásica }\end{array}$ & $\begin{array}{l}\text { Endoscopia } \\
\text { y/o RX }\end{array}$ & $\begin{array}{l}\text { Éxito en la } \\
\text { inserción }\end{array}$ & $\begin{array}{l}\text { Resolución de } \\
\text { la obstrucción } \\
\text { colónica }\end{array}$ & Evolución \\
\hline
\end{tabular}

\begin{tabular}{|c|c|c|c|c|c|c|c|}
\hline 1 & 62 & M & Recto-sigma & Mixta & Sí & Sí & Neo ovárica, paliativo definitivo \\
\hline 2 & 70 & M & Sigma-descendente & Mixta & Sí & Sí & Nueva prótesis para fístula \\
\hline 3 & 70 & $\mathrm{H}$ & Sigma & Mixta & Sí & Sí & Cirugía programada \\
\hline 4 & 87 & M & Recto & Mixta & Sí & Sí & Migró a los 3 meses \\
\hline 5 & 64 & $\mathrm{H}$ & Sigma & Mixta & Sí & Sí & Migró a los 3 días, nueva prótesis \\
\hline 6 & 58 & $\mathrm{H}$ & Recto-sigma & Mixta & Sí & Sí & Cirugía programada \\
\hline 7 & 43 & $\mathrm{H}$ & Recto-sigma & Mixta & Sí & Sí & Paliativo definitivo \\
\hline 8 & 71 & $\mathrm{H}$ & Sigma & Mixta & Sí & Sí & Cirugía programada \\
\hline 9 & 80 & $\mathrm{H}$ & Recto-sigma & Endoscopia sólo & Sí & Sí & Cirugía programada \\
\hline 10 & 83 & M & Sigma & Endoscopia sólo & Sí & $\begin{array}{l}\text { Sí, tránsito intes- } \\
\text { tinal alterado por } \\
\text { carcinomatosis } \\
\text { peritoneal }\end{array}$ & Paliativo definitivo \\
\hline 11 & 79 & $\mathrm{H}$ & Recto & Endoscopia sólo & Sí & Sí & Cirugía programada \\
\hline 12 & 76 & $M$ & Recto-sigma & Mixta & Sí & Sí & Cirugía programada \\
\hline 13 & 77 & $\mathrm{H}$ & Sigma-descendente & Mixta & No & No & Cirugía urgente \\
\hline
\end{tabular}

\section{RESULTADOS}

Durante un período de 18 meses se ha intentado resolver una obstrucción colónica de origen tumoral en 13 pacientes $(8$ hombres, 5 mujeres; edad media 71 años (DE 12), rango 4387) mediante la inserción de PMA. Las prótesis se insertaron satisfactoriamente en 12 pacientes $(92,3 \%)$, obteniéndose la resolución de la obstrucción. La evolución y características de los pacientes se describen en la figura 2 y en la tabla I. En una paciente (número 10 de la tabla I), la obstrucción aguda se resolvió pero el tránsito intestinal estaba alterado a causa de una carcinomatosis peritoneal. No obstante, tres meses después de la inserción de la prótesis, en el momento de redacción de este manuscrito, la paciente sigue viva y está siendo tratada mediante cuidados paliativos. Tres de las prótesis (pacientes 9,10 y 11) se insertaron exclusivamente por medio de endoscopia, sin radiología.

En un paciente (número 13) la guía no pasó a través del tumor, por lo que la prótesis no pudo insertarse. Fue intervenido horas más tarde, encontrándose un gran tumor velloso engastado en el ángulo entre el sigma y el colon descendente.

No hubo complicaciones inmediatas ni mortalidad deriva-

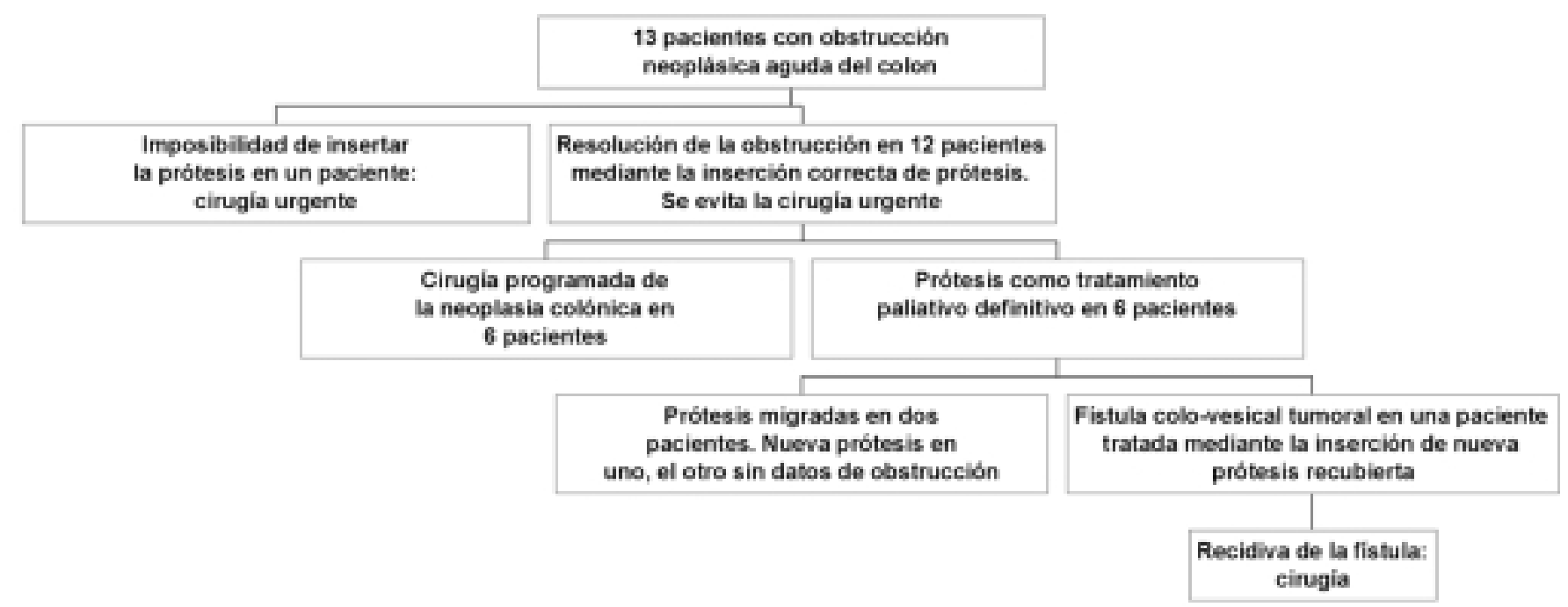

Fig. 2. Evolución de los 13 pacientes en los que se intentó el tratamiento inicial de la obstrucción colónica mediante prótesis metálicas autoexpansibles. 


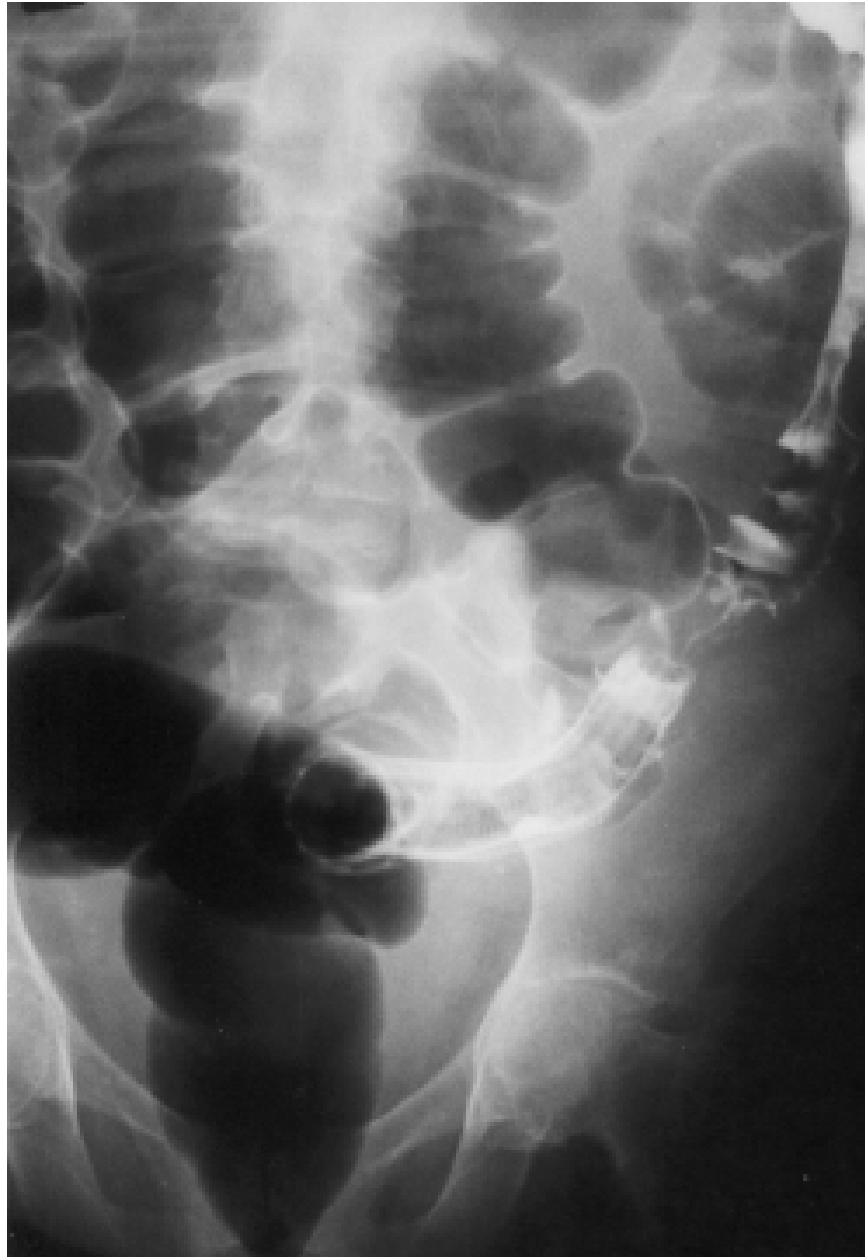

Fig. 3. Se observan en el sigma más proximal y descendente las dos primeras prótesis solapadas para tratar una obstrucción neoplásica (paciente número 2 de la tabla I). Posteriormente desarrolló una fístula colovesical tumoral. Para tratarla se insertó dentro de las dos prótesis anteriores otra, de $14 \mathrm{~cm}$, con malla recubierta (Ultraflex). Esta última prótesis se utiliza habitualmente para las neoplasias de esófago.

da de la inserción de las PMA. Por la extensión de la estenosis tumoral, dos pacientes ( 1 y 2 de la tabla I) necesitaron la colocación de dos prótesis solapadas.

En 6 de los 12 pacientes, tras el estudio de la extensión tumoral, se realizó cirugía programada antes de los 21 días de la inserción de la PMA. Otros seis pacientes (una de ellas con metástasis colónicas de un cáncer ovárico) no fueron considerados aptos para cirugía y la PMA se consideró el tratamiento definitivo. Migraron dos prótesis (17\%, pacientes 4 y 5 de la tabla I). Sólo uno de ellos (paciente 5) necesitó una nueva prótesis. La otra paciente (número 4) no presentó más signos de obstrucción colónica. Esto podría deberse a la dilatación del tumor realizada por la prótesis durante los meses de permanencia in situ.

Una paciente (número 2 de la tabla I) presentaba, desde el primer momento, una tumoración obstructiva que invadía la vejiga urinaria. Tras insertar dos PMA solapadas, ya que la estenosis era mayor de $9 \mathrm{~cm}$, se resolvió la obstrucción. Dos meses después presentó fecaluria en infección urinaria secundarias a una fístula colo-vesical. Fue muy difícil demostrar radiológicamente el paso de contraste del colon a la vejiga, pero, finalmente, se observó que la fístula se encontraba en el centro de las prótesis insertadas anteriormente. Se colocó una PMA recubierta (ultraflex esofágica), con lo que remitió el cuadro (Fig. 3). Sin embargo, mes y medio después la fístula reapareció. La paciente fue intervenida falleciendo en el postoperatorio.

\section{DISCUSIÓN}

La obstrucción aguda del colon forma parte del síndrome de abdomen agudo y debe resolverse con urgencia. Tradicionalmente, la única opción de tratamiento era la cirugía.

La morbimortalidad de los pacientes intervenidos por obstrucción colónica aguda es elevada. Uno de los factores que más influye en las complicaciones de la cirugía es la deficiente preparación del colon. Los pacientes suelen tener una retención importante de heces que conlleva, con frecuencia, la contaminación del campo quirúrgico. Además, con la distensión progresiva del intestino grueso, se producen bacteriemias. El sigma es el lugar anatómico donde con más frecuencia se localiza la obstrucción. Las causas más frecuentes son los tumores y procesos benignos como la diverticulitis.

El procedimiento quirúrgico habitual suele ser una colostomía de descarga, en primer lugar, y meses después, si es posible, la reconstrucción del tránsito colónico normal.

Sin embargo, el enfoque terapéutico de la obstrucción colónica aguda ha cambiado progresivamente desde que, en 1991, se describió, por primera vez, la utilización de PMA para paliar la obstrucción (4). En un estudio reciente de Martínez Santos y cols. (9), del Hospital de Getafe, se comparan el tratamiento quirúrgico tradicional de la obstrucción colónica tumoral, con el de la inserción de PMA. En los pacientes en que la obstrucción se resolvió inicialmente con PMA y se realizó cirugía programada posterior, se ha demostrado una menor incidencia de complicaciones graves y una disminución de la estancia hospitalaria. Además, en el 39\% (17 de 43) de los pacientes en que se insertó una PMA, el estudio de extensión recomendó no operar. La prótesis se consideró como tratamiento paliativo definitivo y se evitaron, por tanto, las intervenciones quirúrgicas.

En la tabla II se muestran algunos trabajos que reflejan la experiencia creciente de la inserción endoscópica de PMA. Como se ve, la tasa media de PMA insertadas por mes, varía entre 0,35 (una prótesis cada tres meses) y 1,4 (algo más de una prótesis al mes). La experiencia actual en nuestro centro, con una tasa de 0,72 , está en un punto intermedio (algo menos de una prótesis al mes). Sin embargo, refleja la utilización creciente de las PMA para tratar la obstrucción colónica, ya que mientras que la mayoría de los trabajos publicados están realizados en grandes centros, nuestro Hospital se encuadra en el nivel II dentro del Sistema Nacional de Salud.

Las PMA utilizadas en el colon, al estar plegadas, caben dentro del canal de trabajo de los endoscopios terapéuticos. La inserción endoscópica de PMA, dentro de la dificultad que toda técnica de intervencionismo comporta, nos parece un procedimiento más sencillo que, por ejemplo, la CPRE (21) y con una curva de aprendizaje más corta. De todos modos, pensamos que para obtener unas tasas de éxito aceptables (superiores en general al 90\% -tabla II-) es preciso tener una dedicación especial. Se requieren tambien unas habilidades distintas de las de la endoscopia diagnóstico-terapéutica con- 


\begin{tabular}{|c|c|c|c|c|c|c|}
\hline \multicolumn{7}{|c|}{ TABLA II } \\
\hline $\begin{array}{l}\text { Autor } \\
\text { (referencia) }\end{array}$ & $\begin{array}{c}\text { Año de } \\
\text { publicación }\end{array}$ & $\begin{array}{l}\text { Número } \\
\text { de PMA }\end{array}$ & $\begin{array}{c}\text { Meses de duración } \\
\text { del estudio }\end{array}$ & $\begin{array}{c}\text { Media de PMA } \\
\text { por cada mes }\end{array}$ & $\begin{array}{l}\text { Porcentaje de inserción } \\
\text { correcta de PMA }\end{array}$ & Observaciones \\
\hline Rey (10) & 1995 & 12 & - & - & $11 / 12(92 \%)$ & Sólo inoperables + láser \\
\hline Saida (11) & 1996 & 15 & & & $12 / 15(80 \%)$ & \\
\hline Dohmoto (12) & 1997 & 19 & - & - & $19 / 19(100 \%)$ & $\begin{array}{l}\text { Sólo inoperables + láser. } \\
\text { Ocho prótesis plásticas }\end{array}$ \\
\hline Tack (13) & 1998 & 10 & - & - & $9 / 10(90 \%)$ & Sólo inoperables + láser \\
\hline Baron (5) & 1998 & 25 & 29 & 0,86 & $23 / 25(92 \%)$ & \\
\hline Adamsen (14) & 2000 & 12 & - & - & $9 / 12(75 \%)$ & $\begin{array}{l}\text { Sólo inoperables, } \\
\text { prótesis recubiertas }\end{array}$ \\
\hline Repici (15) & 2000 & 16 & 20 & 0,8 & $15 / 16(93 \%)$ & PMA recubiertas \\
\hline Lieberman (16) & 2000 & 12 & 33 & 0,36 & $10 / 12(83 \%)$ & \\
\hline Law (17) & 2000 & 24 & 17 & 1,4 & $23 / 24(96 \%)$ & \\
\hline Spinelli (6) & 2001 & 37 & 100 & 0,37 & $36 / 37(97 \%)$ & Sólo inoperables \\
\hline Montes López (18) & 2001 & 24 & 48 & 0,5 & $23724(96 \%)$ & $\begin{array}{l}\text { La mitad insertadas } \\
\text { sólo por radiología }\end{array}$ \\
\hline Ben Sousan (19) & 2001 & 17 & 19 & 0,9 & $16 / 17(94 \%)$ & Sólo tumores inoperables \\
\hline Xinopoulos (20) & 2002 & 11 & 30 & 0,35 & $10 / 11(91 \%)$ & $\begin{array}{l}\text { Sólo tumores } \\
\text { inoperables + láser }\end{array}$ \\
\hline Presente estudio & 2003 & 13 & 18 & 0,72 & $12 / 13(92 \%)$ & \\
\hline
\end{tabular}

Algunos trabajos publicados que tratan sobre la inserción endoscópica de PMA (prótesis metálicas autoexpandibles) para el tratamiento de la obstrucción colónica neoplásica. Sólo se han incluido los que aportan, al menos diez pacientes.

vencional.

Las complicaciones son pocas (22), principalmente la perforación colónica -que necesita una intervención urgente y lógicamente en muy malas condiciones-, la hemorragia y el desplazamiento o migración. Si la prótesis se obstruye por crecimiento tumoral puede recanalizarse, insertando, por dentro, otra prótesis. Lo mismo ocurre en el caso de que migre. No obstante, como ocurrió en una de nuestras pacientes, la dilatación del tumor realizada por la prótesis impide, a veces, la reaparición de un síndrome obstructivo, a pesar de haber migrado, y no siempre es preciso insertar una nueva.

El tratamiento de las fístulas colónicas es otra indicación de las PMA. En este caso deben ser recubiertas. Sin embargo, cuando son de origen tumoral, como en nuestra paciente, frecuentemente recidivan si la supervivencia del enfermo es larga y el tumor crece (23).

\section{Bibliografía}

1. Morice M-C, Serruys PW, Sousa JE, et al. A randomized comparison of a sirolimus-eluting stent with a standard stent for coronary revascularization. N Engl J Med 2002; 346: 1773-80.

2. Slonim SM, Razavi M, Kee S, Semba CP, Dake MD. Transbronchial Palmaz stent placement for tracheo-bronchial stenosis. J Vasc Interv Radiol 1998; 9:153-60.

3. Baron TH. Expandable metal stents for the treatment of cancerous obstructions of the gastrointestinal tract. New Engl J Med 2001; 344: 16811687.

4. Dohmoto M. New method: endoscopic implantation of rectal stent in palliative treatment of malignant stenosis. Endosc Dig 1991; 3: 15071512.

5. Baron TH, Dean PA, Yates MR, et al.Expandable metal stents for the treatment of colonic obstruction: technical outcomes. Gastrointest Endosc 1998; 47: 277-285.
En resumen, las PMA colónicas permiten recanalizar la obstrucción aguda, restableciendo el tránsito intestinal por medio de una técnica mínimamente invasiva. Si el paciente es operable (estadío tumoral en los cánceres, riesgo quirúrgico asumible, etc.) la intervención se puede realizar en buenas condiciones (colon preparado, ausencia de toxemia aguda...) y en un solo tiempo, sin necesidad de colostomía. Si el paciente es inoperable, la prótesis sirve para paliar, de forma definitiva, la obstrucción.

Por la buena experiencia obtenida en este y otros trabajos anteriores, la inserción de prótesis metálicas autoexpandibles podría considerarse, en general, como el tratamiento inicial de la obstrucción colónica a nivel de recto-sigmadescendente. Habría, pues, que reorganizar los recursos sanitarios, para que los pacientes puedan beneficiarse en el momento que lo necesiten de la inserción de PMA por equipos con experiencia.
6. Spinelli P, Mancini A. Use of self-expanding metal stents for palliation of rectosigmoid cancer. Gastrointest Endosc 2001; 53: 203-206.

7. Camuñez F, Echenagusia A, Simo G, et al. Malignant colorectal obstruction treated by means of self-expanding metallic stents:effectiveness before surgery and in palliation. Radiology 2000; 216: 492-497.

8. Mainar A, de Gregorio Ariza MA, Tejero E, et al. Acute colorectal obstruction: treatment with self-expandable metallic stents before scheduled surgery-results of a multicenter study. Radiology 1999; 210: 65-69.

9. Martínez-Santos C, Lobato RF, Fradejas JM, et al. Self-expandable stent before elective surgery vs. emergency surgery for the treatment of malignant colorectal obstructions: comparison of primary anastomosis and morbidity rates. Dis Colon Rectum 2002; 45: 401-406.

10. Rey JF, Romanczyk T, Greff M. Metal stents for palliation of rectal carcinoma: a preliminary report on 12 patients. Endoscopy 1995; 27: 501504. 
11. Saida Y, Sumiyama Y, Nagao J, et al. Stents endoprosthesis for obstructing colorectal cancers. Dis Col Rectum 1996; 39: 552-555.

12. Dohmoto M, H Nerbein M, Schlag M.Application of rectal stents for palliation of obstructing rectosigmoid cancer.Surg Endosc 1997; 11: 758-761.

13. Tack J, Gevers AM, Rutgeerts P. Self-expandable metallic stents in the palliation of rectosigmoidal carcinoma: a follow-up study.Gastrointest Endosc 1998; 48: 267-271.

14. Adamsen S, Holm J, Meisner S, et al.Endoscopic placement of selfexpanding metal stents for treatment of colorectal obstruction with long term follow-up. Dan Med Bull 2000; 47: 225-227.

15. Repici A, Reggio D, DeAngelis C, et al.Covered metal stents for management of inoperable malignant colorectal strictures.Gastrointest Endosc 2000; 52: 735-740,

16. Lieberman H, Adams DR, Blatchford GJ, et al. Clinical use of the selfexpanding metallic stent in the management of colorectal cancer. Am J Surg 2000; 180: 407-411.

17. Law WL, Chu KW, Ho JWC et al. Self-expanding metallic stent in the treatment of colonic obstruction caused by advanced malignancies. Dis
Colon Rectum 2000; 43: 1522-1527.

18. Montes Lopez C, Martinez JMR, Cebrian ET, et al.Treatment of left colon neo-plastic obstruction by placement of self-expandable stents. Rev Esp Enferm Dig 2001; 93: 232-237.

19. Ben Sousan E, Savoye G, Hochain P, et al. Expandable metal stents in palliative treatment of malignant colorectal stricture. A report of 17 consecutive patients. Gastroenterol Clin Biol 2001; 25: 463-467.

20. Xinopoulos D, Dimitroulopoulos D, Tsamakidis K, et al. Treatment of malignant colonic obstructions with metal stents and laser. Hepatogastroenterology 2002; 49: 359-362.

21. García-Cano Lizcano J, González Martín JA. Adiestramiento en la canulación de la vía biliar mediante Colangiopancreatografía retrógrada endoscópica (CPRE). Gastroenterol Hepatol 2000; 23: 404405.

22. Keymling M. Colorectal stenting. Endoscopy 2003; 35: 234-238.

23. Repici A, Reggio D, Saracco G, et al. Self-expanding covered esophageal Ultraflex stent for palliation of malignant colorectal anastomotic obstruction complicated by multiple fistulas. Gastrointest Endosc 2000; 51: 346-348. 